\title{
SÍFILIS ADQUIRIDA NO NORTE DO BRASIL: PREVALÊNCIA DE CASOS DA DOENÇA NA REGIÃO ENTRE 2015 E JUNHO DE 2019
}

\author{
Gabriel da Silva Duarte ${ }^{1}$, Marcelle Thaiany Leal da Silva ${ }^{1}$, Thasmyr das Mercês Gonçalves \\ Corrêa $^{1}$, Alice Nayara Monteiro Navarro ${ }^{1}$, Sarah Andrade Toledo ${ }^{1}$, Emilly Galvão de Almeida ${ }^{1}$. \\ ${ }^{1}$ Universidade Federal do Pará \\ E-mail para correspondência: duartesilvagabriel8@gmail.com
}

Submetido em: 09/10/2020 e aprovado em: 02/08/2021

\section{RESUMO}

Introdução: s casos de sífilis adquirida aumentaram no Brasil e, na região Norte, por isso este estudo teve como objetivo verificar a prevalência de casos no Norte do Brasil entre janeiro de 2015 e junho de 2019. Métodos: realizou-se um estudo quantitativo, retrospectivo, com dados extraídos do site da Secretaria de Vigilância em Saúde, vinculada ao Ministério da Saúde. Resultados e discussão: os resultados obtidos apontam que a sífilis adquirida apresentou aumento entre 2015 e 2018, afetando sobretudo a parcela masculina da sociedade. Conclusão: Diante desse cenário, os órgãos de saúde precisam adotar medidas efetivas para conter a disseminação da sífilis adquirida na região Norte.

Palavras-chave: sífilis, doenças sexualmente transmissíveis, saúde pública.

\begin{abstract}
Introduction: cases of acquired syphilis increased in Brazil and in the North region, so this study aimed to verify the prevalence of cases in Northern Brazil between January 2015 and June 2019. Methods: a quantitative study was carried out, retrospective, with data extracted from the website of the Health Surveillance Secretariat, linked to the Ministry of Health. Results and discussion: the results obtained show that acquired syphilis increased between 2015 and 2018 , mainly affecting the male portion of society. Conclusion: Given this scenario, health agencies need to adopt effective measures to contain the spread of syphilis acquired in the North region.
\end{abstract}

Keywords: syphilis, sexually transmitted diseases, public health.

\section{INTRODUÇÃO}

A sífilis é uma infecção sexualmente transmissível que, na era contemporânea, representa uma problemática séria na saúde pública brasileira. Causada pela bactéria Treponema pallidum, a doença se faz presente no contexto da saúde global e cada vez mais torna-se motivo de grande preocupação. Anualmente, estima-se que 12 milhões de novos casos de sífilis sejam registrados em todo o mundo ${ }^{(1)}$.

A sífilis adquirida apresentou avanço no Brasil na última década e é preciso que ocorra o debate em torno dessa questão ${ }^{(2,3)}$. 
Na região Norte do Brasil, historicamente marcada por processos de desigualdade de políticas públicas em relação ao contexto nacional, viu-se o crescimento dos casos de sífilis adquirida $^{(4,5)}$. Aliado a essa realidade, sabe-se que o Norte do país recebe menor quantidade de recursos para a saúde, poucas políticas de assistência, bem como possui um quantitativo de profissionais da saúde baixo para as demandas da região ${ }^{(6)}$, fato que reforça o alerta para a situação da saúde e o enfrentamento aos casos de sífilis adquirida.

Este estudo teve como objetivo verificar a prevalência de casos de sífilis adquirida na região Norte do Brasil, entre janeiro de 2015 e junho de 2019.

\section{MÉTODOS}

Realizou-se uma pesquisa a partir da coleta dos dados epidemiológicos disponíveis na plataforma online "Indicadores e Dados Básicos da Sífilis nos Municípios Brasileiros", vinculada ao Ministério da Saúde, sob a regência da Secretaria de Vigilância em Saúde. Assim, realizou-se um recorte temporal específico, sendo, portanto, um estudo de característica retrospectiva, utilizando os dados registrados que se referem ao período compreendido entre janeiro de 2015 até junho de 2019.

Os dados foram tabulados utilizando o programa Excel®. Por ser um estudo em que há apenas a análise de dados, não houve necessidade de submissão ao Comitê de Ética em Pesquisa, conforme a Resolução 510/2016, do Conselho Nacional de Saúde.

\section{RESULTADOS E DISCUSSÃO}

A partir de 2015, houve um aumento no número de casos, em todas as regiões do Brasil, até o ano de 2018, tendo em seguida uma redução até junho de 2019. Seguindo a tendência nacional, o Norte acompanha o fenômeno de crescimento (figura 1).

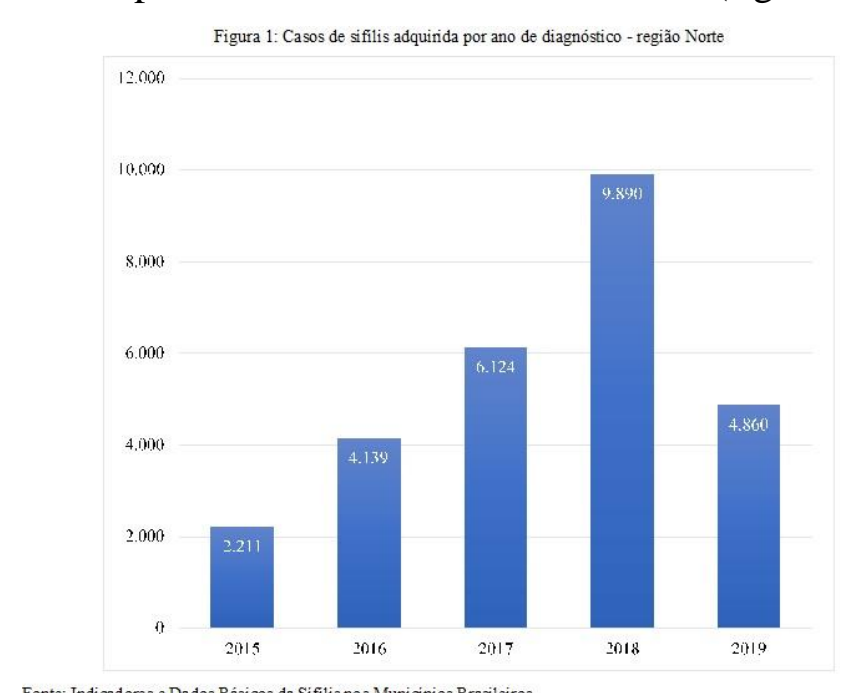


No ano de 2018, foram registrados 9.890 casos (5.736 em homens e 4.154 em mulheres), apresentou a taxa de detecção em 54,4 casos por 100.000 habitantes. Foi o ano com maior incidência no período analisado (Figura 2) ${ }^{(11)}$.

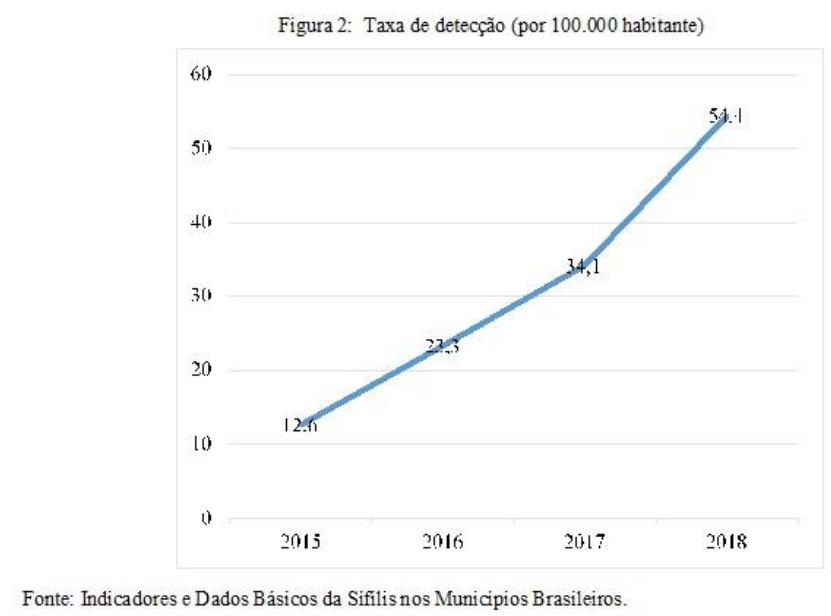

Os casos na região Norte passaram de 2.211 notificações no ano de 2015 para 9.890 em 2018, um aumento de mais de $300 \%^{(8)}$. Alguns fatores acabam por contribuir para isso, tais como as práticas sexuais inseguras aliadas ao elevado número de parceiros/parceiras sexuais $^{(9,12,13)}$. Além desse aspecto, outra leitura pode ser feita: há o aumento do número de pessoas mantendo relações sexuais de forma insegura ${ }^{(14)}$. Revela-se, portanto, que as condutas sexuais perigosas são o principal impulsionador para o crescimento de casos na região Norte e no Brasil como um todo.

O fato de não haver uma exatidão no surgimento do primeiro sintoma faz com que um indivíduo que mantenha relações sexuais com diversos parceiros/parceiras, sem saber que está infectado, eleve a chance de contágio pela sífilis ${ }^{(15,16)}$. A subnotificação dos casos da doença é recorrente. Muitas pessoas infectam-se, mas não manifestam sintomas ${ }^{(17)} \mathrm{e}$, dificultam desta forma o diagnóstico.

No período estudado, os casos de sífilis adquirida são maiores entre o sexo masculino (figura 3). A resposta mais aceita para que isso ocorra é o fato de os homens terem maior resistência à utilização de preservativos. Diversos estudos ${ }^{(18,19,20)}$, corroboram essa ideia e despertam o alerta para a necessidade de conscientização por partes dos órgãos de saúde para que o gênero masculino, sobretudo a parcela mais jovem, sobre a necessidade e importância de preservativos. 


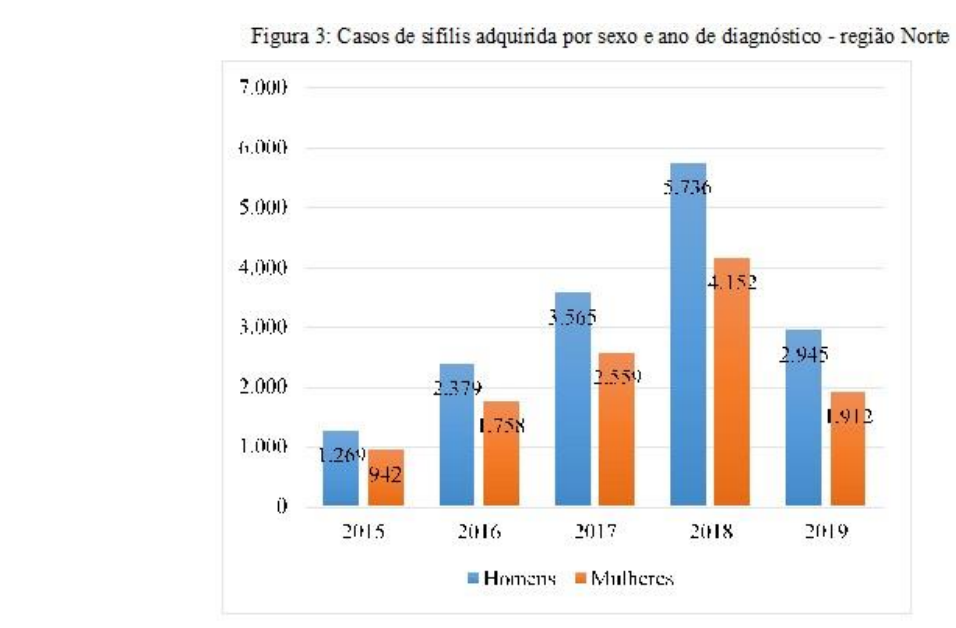

Fonte: Indicadores e Dados Básicos da Sifilis nos Municipios Brasileiros.

\section{CONCLUSÃO}

Diante desse cenário, os órgãos de saúde precisam adotar medidas efetivas para conter a disseminação da sífilis adquirida na região Norte.

\section{REFERÊNCIAS BIBLIOGÁFICAS}

1. Conceição, H. N. D., Câmara, J. T., \& Pereira, B. M. (2020). Análise epidemiológica e espacial dos casos de sífilis gestacional e congênita. Saúde em debate, 43, 1145-1158.

2. Santos SB, Machado APA, Sampaio LA et. al. Sífilis adquirida: construção e validação de tecnologia educativa para adolescentes. J Hum Growth Dev. 2019; 29(1): 65-74.

3. BRASIL. Ministério da Saúde. Secretaria de Vigilância em Saúde. Boletim Epidemiológico da Sífilis, v. 47, n. 35, 2016. Disponível em: http://www.aids.gov.br/pt-br/pub/2018/boletimepidemiologico-de-sifilis-2016.

4. Saraceni V, Pereira GFM, Silveira MF et al. Vigilância epidemiológica da transmissão vertical da sífilis: dados de seis unidades federativas no Brasil. Rev Panam Salud Publica. 2017;41:e44.

5. BRASIL. Ministério da Saúde. Secretaria de Vigilância em Saúde. Boletim Epidemiológico da Sífilis, v. 50, Número especial, 2020. Disponível em: http://www.aids.gov.br/ptbr/pub/2020/boletim-sifilis-2020.

6. Garnelo L, Lima JG, Rocha ESC et al. Acesso e cobertura da Atenção Primária à Saúde para populações rurais e urbanas na região norte do Brasil. SAÚDE DEBATE. RIO DE JANEIRO, V. 42, NÚMERO ESPECIAL 1, P. 81-99, 2018.

7. BRASIL. Ministério da Saúde. Secretaria de Vigilância em Saúde. Indicadores e dados básicos da sífilis nos municípios brasileiros. Acesso em: 05 de agosto de 2020. Disponível em: http://indicadoressifilis.aids.gov.br/.

8. BRASIL. Ministério da Saúde. Secretaria de Vigilância em Saúde. Boletim Epidemiológico da Sífilis, v. 49, Número especial, 2019. Disponível em: http://www.aids.gov.br/ptbr/pub/2018/boletim-epidemiologico-de-sifilis-2019. 
9. Arando LM, Otero GL. Sífilis. Enferm Infecc Microbiol Clin. 2019. https://doi.org/10.1016/j.eimc.2018.12.009.

10. Santos LG, Dantas ASC, Santos LFS et al. As diversidades da predominância da sífilis adquirida nas regiões do Brasil (2010 - junho de 2019). Revista Eletrônica Acervo Científico / Electronic Journal Scientific Collection | Vol. $10 \mid$ e3553 | DOI: https://doi.org/10.25248/reac.e3553.2020.

11. Chiacchio, AD. Escobar, ND. Gilo, NF. et al. Perfil epidemiológico de sífilis adquirida nas regiões do Brasil no período de 2010 a 2019. Revista Amazônia Science \& Health 2020, Vol. 8, $\mathrm{N}^{\mathrm{o}}$ 2. DOI 10.18606/2318-1419/amazonia.sci.health.v8n2p51-63

12. Dias, A. P. D. S. L., de Melo Wanzeller, R. C., Vital, R. D. S. S., \& da Silveira, A. D. P. S. (2018). A sífilis no atual cenário brasileiro. Health Research Journal, 1(2), 1-21.

13. Silva EC, Tupinambá MR, Silva FASD et al. Resultados de sorologia para casos de sífilis em campanha de município no norte do Brasil. Rev Pan-Amaz Saude 2016; 7(1):39-43. http://revista.iec.pa.gov.br.

14. Morgado-Carrasco D, Gibert MA, Mestres JB et al. Infecciones de transmisión sexual con afectación anorrectal: agentes causales, coinfecciones, infección por el VIH y conductas de riesgo. Med Clin (Barc). 2017. https://doi.org/10.1016/j.medcli.2018.02.014.

15. Araújo MAL, Rocha AFB, Cavalcante EGF et al. Doenças sexualmente transmissíveis atendidas em unidade primária de saúde no Nordeste do Brasil. Cad. Saúde Colet., 2015, Rio de Janeiro, 23 (4): 347-353.

16. Freitas GM. Notificação da sífilis adquirida em uma Superintendência Regional de Saúde do sul de Minas Gerais. Alfenas/MG: Universidade Federal de Alfenas - UNIFAL-MG, 2018. Dissertação de Mestrado em Enfermagem.

17. Ros-Vivancos C, González-Hernández M, Navarro-Gracia JF et al. Evolución del tratamiento de la sífilis a lo largo de la historia. Rev Esp Quimioter 2018;31(6): 485-492.

18. Andrews, C.H., Faxelid, E., Sychaerun, V. et al. Determinants of consistent condom use among female sex workers in Savannakhet, Lao PDR. BMC Women's Health 15, 63 (2015). https://doi.org/10.1186/s12905-015-0215-0.

19. Barbosa KF, Batista AP, Nacife MBPSL et al. Fatores associados ao não uso de preservativo e prevalência de HIV, hepatites virais B e C e sífilis: estudo transversal em comunidades rurais de Ouro Preto, Minas Gerais, entre 2014 e 2016. Epidemiol. Serv. Saude, Brasília, 28(2):e2018408, 2019. https://doi.org/10.5123/S1679-49742019000200023.

20. Soares ES, Carvalho EM, Lima KTLL. Incidência de sífilis adquirida em uma cidade da microrregião do sudoeste baiano. Rev. Bras. Anal. Clin., Rio de Janeiro, 2019, ISSN (online): 2448-3877. https://doi.org/10.21877/2448-3877.201900757. 